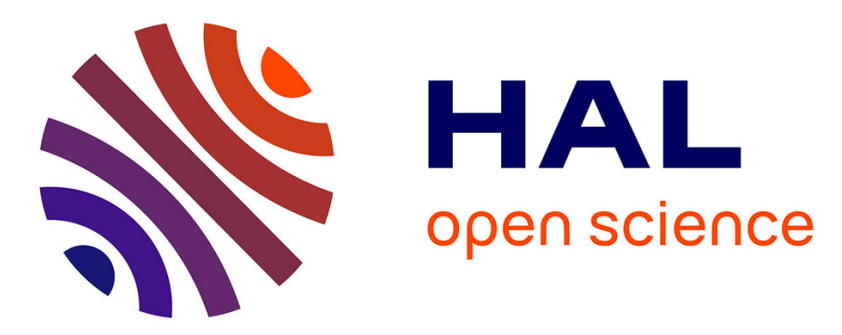

\title{
A fully decoupled scheme for the interaction of a thin-walled structure with an incompressible fluid
}

Miguel Angel Fernández, Mikel Landajuela

\section{To cite this version:}

Miguel Angel Fernández, Mikel Landajuela. A fully decoupled scheme for the interaction of a thinwalled structure with an incompressible fluid. Comptes Rendus. Mathématique, 2013, 351 (3-4), pp.161-164. 10.1016/j.crma.2013.02.015 . hal-00795585

\section{HAL Id: hal-00795585 \\ https://hal.inria.fr/hal-00795585}

Submitted on 28 Feb 2013

HAL is a multi-disciplinary open access archive for the deposit and dissemination of scientific research documents, whether they are published or not. The documents may come from teaching and research institutions in France or abroad, or from public or private research centers.
L'archive ouverte pluridisciplinaire HAL, est destinée au dépôt et à la diffusion de documents scientifiques de niveau recherche, publiés ou non, émanant des établissements d'enseignement et de recherche français ou étrangers, des laboratoires publics ou privés. 


\title{
A fully decoupled scheme for the interaction of a thin-walled structure with an incompressible fluid ${ }^{1}$
}

\author{
Miguel A. Fernández ${ }^{\mathrm{a}, \mathrm{b}}$, Mikel Landajuela ${ }^{\mathrm{a}, \mathrm{b}}$ \\ ${ }^{a}$ Inria, REO team, Rocquencourt - B.P. 105, F-78153 Le Chesnay cedex, France \\ ${ }^{\mathrm{b}}$ UPMC Univ Paris VI, REO team, UMR 7958 LJLL, F-75005 Paris, France \\ Received $* * * * * ;$ accepted after revision +++++ \\ Presented by
}

\begin{abstract}
In this note we propose a class of fully decoupled schemes (velocity-pressure-displacement splitting) for the coupling of an incompressible fluid with a thin-walled structure. The time splitting is achieved by combining an overall fractional-step time-marching of the system with a specific Robin-Neumann treatment of the interface coupling. The two variants considered yield unconditional stability. Numerical experiments in a benchmark show that, for one of them, the splitting does not compromises the optimal convergence rate. To cite this article: A.Name1, A. Name2, C. R. Acad. Sci. Paris, Ser. I 340 (2005).

\section{Résumé}

Un schéma totalement découplée pour l'interaction d'une structure mince avec un fluide incompressible. Dans cette note, nous proposons un type de schémas totalement découplés (vitesse-pression-déplacement) pour le couplage d'un fluide incompressible avec une structure mince. Le découplage en temps est obtenu en combinant un schéma à pas fractionnaire sur l'ensemble du système avec un traitement spécifique Robin-Neumann des conditions d'interface. Les deux variantes considérées sont inconditionnellement stables. Des expriences numériques montrent que, pour l'une d'elles, on obtient un taux de convergence optimal. Pour citer cet article: A. Name1, A. Name2, C. R. Acad. Sci. Paris, Ser. I 340 (2005).
\end{abstract}

Email addresses: miguel.fernandez@inria.fr (Miguel A. Fernández), mikel.landajuela_arma@inria.fr (Mikel Landajuela).

1. This work has been supported by the french National Research Agency (ANR) through the EXIFSI project (ANR12-JS01-0004). 


\section{Introduction}

Fractional-step schemes have been widely used in incompressible fluid-structure interaction as a way to avoid the infamous added-mass numerical instabilities featured by conventional explicit coupling schemes (which invoke the fluid and solid solvers only once per time-step). The resulting time-marching procedures are semi-implicit in the sense that they combine either a fractional-step method in the fluid $[4,10,1]$ or in the solid $[8,3,6]$ with an implicit-explicit treatment of the interface conditions. These methods guarantee different degrees of fluid-solid splitting in the time-marching, via an explicit treatment of either the fluid viscous contributions or the solid (visco-)elastic contributions, without compromising stability.

In this note, we consider the case of the coupling with a thin-walled structure and propose to go further in the time-splitting of the coupled system by combining both approaches. The resulting schemes enable (for the first time) a fully decoupled computation of the entire fluid-solid state (velocity, pressure, displacement). We present an a priori energy estimate which guarantees (added-mass free) unconditional stability. The accuracy of the methods is illustrated in a numerical benchmark. The results show that one of the variants preserves the optimal convergence rate.

\section{A linear fluid-structure model problem}

We consider a low Reynolds regime and assume that the structure undergoes infinitesimal displacements. The fluid is described by the Stokes equations in a fixed domain $\Omega \subset \mathbb{R}^{d}(d=2,3)$, with boundary partitioned as $\partial \Omega=\Gamma \cup \Sigma$, where $\Sigma$ stands for the fluid-structure interface. The structure is assumed to behave as a linear thin membrane represented by the $(d-1)$-manifold $\Sigma$. The coupled problem reads then as follows: find the fluid velocity $\boldsymbol{u}: \Omega \times \mathbb{R}^{+} \rightarrow \mathbb{R}^{d}$, the fluid pressure $p: \Omega \times \mathbb{R}^{+} \rightarrow \mathbb{R}$, the solid normal displacement $\eta: \Sigma \times \mathbb{R}^{+} \rightarrow \mathbb{R}$ and the solid normal velocity $\dot{\eta}: \Sigma \times \mathbb{R}^{+} \rightarrow \mathbb{R}$ such that

$$
\left\{\begin{array} { r l } 
{ \rho ^ { \mathrm { f } } \partial _ { t } \boldsymbol { u } - \operatorname { d i v } \boldsymbol { \sigma } ( \boldsymbol { u } , p ) = \mathbf { 0 } } & { \text { in } \quad \Omega , } \\
{ \operatorname { d i v } \boldsymbol { u } = 0 } & { \text { in } \quad \Omega , } \\
{ \boldsymbol { \sigma } ( \boldsymbol { u } , p ) \boldsymbol { n } = - p _ { \Gamma } \boldsymbol { n } } & { \text { on } \quad \Gamma , } \\
{ \boldsymbol { u } \cdot \boldsymbol { \tau } = 0 } & { \text { on } \quad \Sigma , }
\end{array} \quad \{ 1 ) \quad \left\{\begin{array}{rll}
\boldsymbol{u} \cdot \boldsymbol{n}=\dot{\eta} & \text { on } & \Sigma, \\
\rho^{\mathrm{s}} \epsilon \partial_{t} \dot{\eta}+L^{\mathrm{v}} \dot{\eta}+L^{\mathrm{e}} \eta=-(\boldsymbol{\sigma}(\boldsymbol{u}, p) \boldsymbol{n}) \cdot \boldsymbol{n} & \text { in } & \Sigma, \\
\dot{\eta}=\partial_{t} \eta & \text { in } & \Sigma, \\
\eta=0 & \text { on } & \partial \Sigma,
\end{array}\right.\right.
$$

complemented with the initial conditions $\boldsymbol{u}(0)=\boldsymbol{u}_{0}, \eta(0)=\eta_{0}$ and $\dot{\eta}(0)=\dot{\eta}_{0}$. Here, $\rho^{\mathrm{f}}$ and $\rho^{\mathrm{s}}$ stand for the fluid and solid densities, $\epsilon$ for the solid thickness, $\varepsilon(\boldsymbol{u}) \stackrel{\text { def }}{=} \frac{1}{2}\left(\boldsymbol{\nabla} \boldsymbol{u}+\boldsymbol{\nabla} \boldsymbol{u}^{\mathrm{T}}\right)$ for the fluid strain rate tensor, $\mu$ for the fluid dynamic viscosity, $\boldsymbol{\sigma}(\boldsymbol{u}, p) \stackrel{\text { def }}{=}-p \boldsymbol{I}+2 \mu \boldsymbol{\varepsilon}(\boldsymbol{u})$ for the fluid Cauchy stress tensor, $p_{\Gamma}$ for a given inlet/outlet pressure and $\boldsymbol{n}$ and $\boldsymbol{\tau}$ for the exterior unit normal and tangent vectors on $\partial \Omega$. The differential operators $L^{\mathrm{e}}$ and $L^{\mathrm{v}}$ describe the viscoelastic behavior of the structure. Though simplified, problem (1)-(2) features some of the main numerical issues that appear in complex non-linear fluid-structure interaction problems involving a viscous incompressible fluid (see, e.g., [2]).

\section{Time semi-discretization: fully decoupled time-marching}

In this section we introduce a time discretization of the coupled problem (1)-(2) which enables a fully decoupled sequential computation of the fluid-solid state $(\boldsymbol{u}, p, \eta)$. The main idea consists in combining an overall fractional-step time-marching in the system with a specific splitting of the fluid-structure interface coupling. The fluid problem (1) is discretized using a standard Chorin-Temam scheme as follows (see, e.g., $[7])$ : 


$$
\left\{\begin{aligned}
\rho^{\mathrm{f}} \frac{\tilde{\boldsymbol{u}}^{n}-\boldsymbol{u}^{n-1}}{\delta t}-2 \mu \operatorname{div} \boldsymbol{\varepsilon}\left(\tilde{\boldsymbol{u}}^{n}\right) & =\mathbf{0} \text { in } \Omega, \\
\tilde{\boldsymbol{u}} \cdot \boldsymbol{\tau} & =0 \text { on } \Sigma,
\end{aligned}\right.
$$

$$
\left\{\begin{aligned}
\rho^{\mathrm{f}} \frac{\boldsymbol{u}^{n}-\tilde{\boldsymbol{u}}^{n}}{\delta t}+\nabla p^{n} & =\mathbf{0} & & \text { in } \quad \Omega, \\
\operatorname{div} \boldsymbol{u}^{n} & =0 & & \text { in } \quad \Omega, \\
p^{n} & =p_{\Gamma} & & \text { on } \quad \Gamma, \\
\boldsymbol{u}^{n} \cdot \boldsymbol{\tau} & =0 & & \text { on } \quad \Sigma .
\end{aligned}\right.
$$

In (2) we perform the following three-step Yanenko splitting, with a specific treatment of the fluid-solid kinematic and kinetic coupling,

$$
\begin{aligned}
& \left\{\begin{array}{r}
\tilde{\boldsymbol{u}}^{n} \cdot \boldsymbol{n}=\dot{\eta}^{n-\frac{1}{3}} \quad \text { on } \quad \Sigma, \\
\rho^{\mathrm{s} \epsilon \frac{\dot{\eta}^{n-\frac{1}{3}}-\dot{\eta}^{n-1}}{\delta t}=-\left(2 \mu \varepsilon\left(\tilde{\boldsymbol{u}}^{n}\right) \boldsymbol{n}\right) \cdot \boldsymbol{n}} \quad \text { in } \quad \Sigma,
\end{array}\right.
\end{aligned}
$$

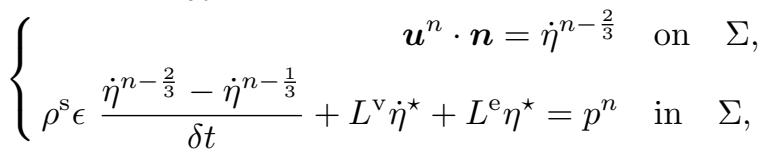

$$
\begin{aligned}
& \left\{\begin{aligned}
\rho^{\mathrm{s}} \epsilon \frac{\dot{\eta}^{n}-\dot{\eta}^{n-\frac{2}{3}}}{\delta t}+L^{\mathrm{v}}\left(\dot{\eta}^{n}-\dot{\eta}^{\star}\right)+L^{\mathrm{e}}\left(\eta^{n}-\eta^{\star}\right)=0 & \text { in } \quad \Sigma, \\
\dot{\eta}^{n}=\frac{\eta^{n}-\eta^{n-1}}{\delta t} & \text { in } \quad \Sigma, \\
\eta^{n}=0 & \text { on } \quad \partial \Sigma .
\end{aligned}\right.
\end{aligned}
$$

The superscript * indicates zeroth- (i.e., without) or first-order extrapolation from the previous time-steps. Hence, the solid displacement and velocity extrapolations considered in (6) and (7) are either $\left(\eta^{\star}, \dot{\eta}^{\star}\right)=\mathbf{0}$ or $\left(\eta^{\star}, \dot{\eta}^{\star}\right)=\left(\eta^{n-1}, \dot{\eta}^{n-1}\right)$.

Some remarks are in order. The traditional velocity/pressure splitting of the Chorin-Temam scheme (3)-(4) is preserved via the splitting of the viscous and pressure contribution of the interface fluid stress in steps (5) and (6). Added-mass free stability is simply guaranteed (see Proposition 3.1 below) by treating implicitly the coupling between the fluid pressure/incompressibility and the solid inertia, that is, by simultaneously solving steps (4) and (6). At last, the extrapolation of the viscoelastic solid contributions, in steps (6) and (7), is performed to control the perturbation of the kinematic coupling, which dramatically affects accuracy (see Section 4 below, and [6]).

The resulting time-marching scheme is detailed in Algorithm 1. The standard 'Neumann' solid sub-step (10) is obtained by simply adding the relations $(5)_{2},(6)_{2}$ and $(7)_{1}$. We have eliminated $\boldsymbol{u}^{n-1}$ in $(3)$ via $(4)_{1}$, and the projection sub-step (4) is reformulated as a pressure-Poisson problem. It is worth noting that, since the structure is thin, the simultaneous solution of (3) and (5) (resp. (4) and (6)) is embedded into the single sub-step (8) (resp. (9)) through explicit Robin conditions. The pressure-Poisson problem (9) has a rather intrinsic nature, independent of the viscoelastic contributions involved in (6). In fact, from the relations $(10)_{1}$ and $(8)_{2}$, we can infer that $L^{\mathrm{v}} \dot{\eta}^{\star}+L^{\mathrm{e}} \eta^{\star}=p^{\star}+\frac{\rho^{\mathrm{s}} \epsilon}{\delta t}\left(\tilde{\boldsymbol{u}}^{\star} \cdot \boldsymbol{n}-\dot{\eta}^{\star}\right)$, which yields a purely algebraic relation in terms of $\tilde{\boldsymbol{u}}^{\star}, p^{\star}$ and $\dot{\eta}^{\star}$, with $\left(\tilde{\boldsymbol{u}}^{\star}, p^{\star}, \dot{\eta}^{\star}\right)=\mathbf{0}$ or $\left(\tilde{\boldsymbol{u}}^{\star}, p^{\star}, \dot{\eta}^{\star}\right)=\left(\tilde{\boldsymbol{u}}^{n-1}, p^{n-1}, \dot{\eta}^{n-1}\right)$. In practice, this means that modifications in the solid equation only affect the solid sub-step in Algorithm 1.

The following result states the energy based stability of Algorithm 1, whose proof can be found in the forthcoming work [5].

Proposition 3.1 Assume that $p_{\Gamma}=0$ (free system). The following a priori energy estimate holds for $n \geq 1$ :

$$
E^{n}+D^{n}< \begin{cases}E^{0} & \text { if } \quad\left(\eta^{\star}, \dot{\eta}^{\star}\right)=(0,0), \\ E^{0}+\frac{\delta t^{2}}{2} \int_{\Sigma} \dot{\eta}^{0} L^{\mathrm{e}} \dot{\eta}^{0}+\frac{\delta t^{2}}{2 \rho^{\mathrm{s}} \epsilon}\left\|L^{\mathrm{v}} \dot{\eta}^{0}+L^{\mathrm{e}} \eta^{0}\right\|_{L^{2}(\Sigma)}^{2} & \text { if } \quad\left(\eta^{\star}, \dot{\eta}^{\star}\right)=\left(\eta^{n-1}, \dot{\eta}^{n-1}\right),\end{cases}
$$


where the total semi-discrete energy and dissipation are defined by

$$
E^{n} \stackrel{\text { def }}{=} \frac{\rho^{\mathrm{f}}}{2}\left\|\boldsymbol{u}^{n}\right\|_{L^{2}(\Omega)}^{2}+\frac{\rho^{\mathrm{s}} \epsilon}{2}\left\|\dot{\eta}^{n}\right\|_{L^{2}(\Sigma)}^{2}+\frac{1}{2} \int_{\Sigma} \eta^{n} L^{\mathrm{e}} \eta^{n}, \quad D^{n} \stackrel{\text { def }}{=} \sum_{m=1}^{n} \delta t\left(2 \mu\left\|\varepsilon\left(\tilde{\boldsymbol{u}}^{m}\right)\right\|_{L^{2}(\Omega)}^{2}+\int_{\Sigma} \dot{\eta}^{m} L^{\mathrm{v}} \dot{\eta}^{m}\right)
$$

Algorithm 1 Time semi-discrete fully decoupled scheme (for (1)-(2))

(i) Fluid viscous sub-step: find $\tilde{\boldsymbol{u}}^{n}: \Omega \rightarrow \mathbb{R}^{d}$ such that

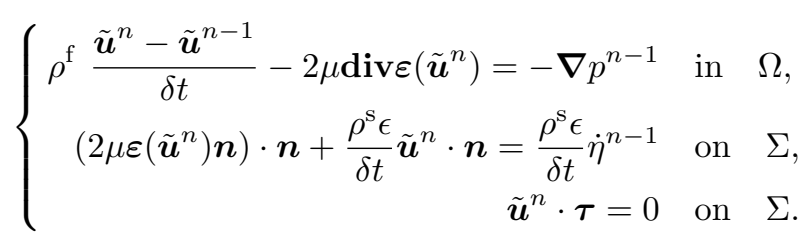

(ii) Fluid projection sub-step (pressure-Poisson formulation): find $p^{n}: \Omega \rightarrow \mathbb{R}$ such that

$$
\left\{\begin{array}{r}
-\frac{\delta t}{\rho^{\mathrm{f}}} \Delta p^{n}=-\operatorname{div}\left(\tilde{\boldsymbol{u}}^{n}\right) \quad \text { in } \quad \Omega, \\
p^{n}=p_{\Gamma} \quad \text { on } \quad \Gamma, \\
\frac{\delta t}{\rho^{\mathrm{f}}} \frac{\partial p^{n}}{\partial n}+\frac{\delta t}{\rho^{\mathrm{s}} \epsilon} p^{n}=\frac{\delta t}{\rho^{\mathrm{s}} \epsilon} p^{\star}+\tilde{\boldsymbol{u}}^{\star} \cdot \boldsymbol{n}-\dot{\eta}^{\star} \quad \text { on } \quad \Sigma .
\end{array}\right.
$$

(iii) Solid sub-step: find $\eta^{n}: \Sigma \rightarrow \mathbb{R}$ and $\dot{\eta}^{n}: \Sigma \rightarrow \mathbb{R}$ such that

$$
\left\{\begin{aligned}
\rho^{\mathrm{s}} \epsilon \frac{\dot{\eta}^{n}-\dot{\eta}^{n-1}}{\delta t}+L^{\mathrm{v}} \dot{\eta}^{n}+L^{\mathrm{e}} \eta^{n}=-\left(\boldsymbol{\sigma}\left(\tilde{\boldsymbol{u}}^{n}, p^{n}\right) \boldsymbol{n}\right) \cdot \boldsymbol{n} & \text { in } \quad \Sigma, \\
\dot{\eta}^{n}=\frac{\eta^{n}-\eta^{n-1}}{\delta t} & \text { in } \Sigma, \\
\eta^{n}=0 & \text { on } \partial \Sigma .
\end{aligned}\right.
$$

\section{Numerical experiment}

In order to illustrate the accuracy of the proposed schemes, we consider the well-known example of the propagation of a pressure-wave within an elastic tube (see, e.g., [3]). We couple the 2D Stokes equations with a $1 \mathrm{D}$ generalized string model, $L^{\mathrm{e}} \eta=-c_{1} \partial_{x x} \eta+c_{0} \eta$ and $L^{\mathrm{v}} \dot{\eta}=-\beta c_{1} \partial_{x x} \dot{\eta}+\alpha \rho^{\mathrm{s}} \epsilon \dot{\eta}$ in (2). The fluid domain is given by the rectangle $\Omega=[0,6] \times[0,0.5]$ and the interface by the segment $\Sigma=[0,6] \times\{0.5\}$, all the units are given in the CGS system. At $x=0$ we impose a sinusoidal pressure of maximal amplitude $2 \times 10^{4}$ during $5 \times 10^{-3}$ seconds, corresponding to half a period. Zero pressure is enforced at $x=6$ and a symmetry condition is applied on the lower wall $y=0$. The fluid physical parameters are given by $\rho^{\mathrm{f}}=1.0, \mu=0.035$. For the solid we have $\rho^{\mathrm{s}}=1.1, \epsilon=0.1, \alpha=1, \beta=10^{-3}, c_{1}=E \epsilon /(2(1+\nu))$ and $c_{0}=E \epsilon /\left(0.25\left(1-\nu^{2}\right)\right)$, with Young's modulus $E=0.75 \times 10^{6}$ and Poisson's ratio $\nu=0.5$. Piece-wise affine continuous finite elements are used for the discretization in space. The computations have been performed with FreeFem $++[9]$.

Figure 1(left) reports the time-convergence history of the solid displacement at time $t=0.015$, in the relative elastic energy-norm, obtained with Algorithm 1 and a fully implicit first-order scheme. The reference solutions have been generated with the implicit scheme using a high space-time resolution. We 

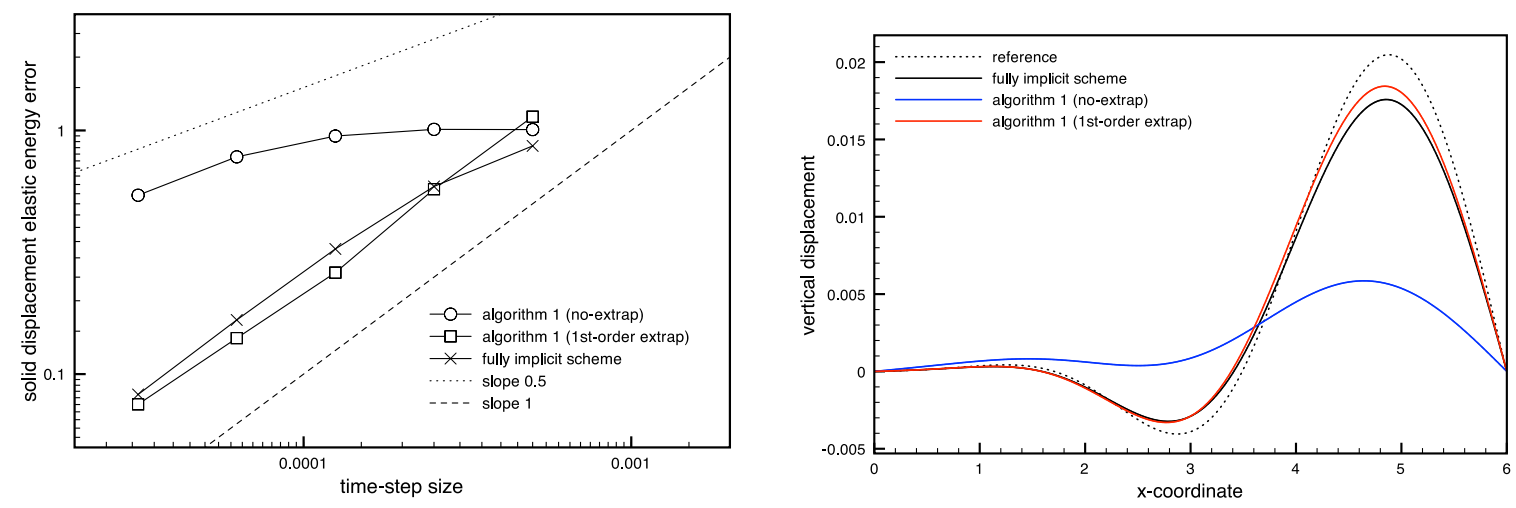

Figure 1. Left: displacement convergence history in time, $h=O(\delta t)$. Right: displacement at time $t=0.015 \mathrm{~s}$.

have refined both in time and in space according to $(\delta t, h)=\left\{5 \cdot 10^{-4} / 2^{i}, 10^{-1} / 2^{i}\right\}_{i=0}^{4}$. In Figure 1(right) we show a comparison of the solid displacements at time $t=0.015$, obtained with $i=3$. The results show that the proposed fully decoupled scheme with first-order extrapolations is able to retrieve the optimal first-order convergence rate of the implicit scheme. A sub-optimal overall rate is obtained without viscoelastic extrapolation.

\section{References}

[1] S. Badia, A. Quaini, and A. Quarteroni. Splitting methods based on algebraic factorization for fluid-structure interaction. SIAM J. Sci. Comput., 30(4):1778-1805, 2008.

[2] P. Causin, J.-F. Gerbeau, and F. Nobile. Added-mass effect in the design of partitioned algorithms for fluid-structure problems. Comput. Methods Appl. Mech. Engrg., 194(42-44):4506-4527, 2005.

[3] M.A. Fernández. Incremental displacement-correction schemes for incompressible fluid-structure interaction: stability and convergence analysis. Numer. Math., 123(1):21-65, 2013.

[4] M.A. Fernández, J.F. Gerbeau, and C. Grandmont. A projection semi-implicit scheme for the coupling of an elastic structure with an incompressible fluid. Int. J. Num. Meth. Engrg., 69(4):794-821, 2007.

[5] M.A. Fernández and M. Landajuela. Fractional-step time-marching schemes for the coupling of incompressible fluids with thin-walled structures. In preparation.

[6] M.A. Fernández, J. Mullaert, and M. Vidrascu. Explicit Robin-Neumann schemes for the coupling of incompressible fluids with thin-walled structures. Research Report RR-8224, Inria, February 2013.

[7] J. L. Guermond, P. Minev, and J. Shen. An overview of projection methods for incompressible flows. Comput. Methods Appl. Mech. Engrg., 195(44-47):6011-6045, 2006.

[8] G. Guidoboni, R. Glowinski, N. Cavallini, and S. Canic. Stable loosely-coupled-type algorithm for fluid-structure interaction in blood flow. J. Comp. Phys., 228(18):6916-6937, 2009.

[9] O. Pironneau, F. Hecht, A. Le Hyaric, and J. Morice. Freefem++, www.freefem.org/ff++.

[10] A. Quaini and A. Quarteroni. A semi-implicit approach for fluid-structure interaction based on an algebraic fractional step method. Math. Models Methods Appl. Sci., 17(6):957-983, 2007. 